-210J. Inv. Ill-Posed Problems, Vol. 8, No. 2, pp. 205-210 (2000)

(c) VSP 2000

\title{
Finding small inhomogeneities from scattering data
}

\author{
A. G. RAMM* \\ Received January 18, 1999
}

\begin{abstract}
A new method for finding small inhomogeneities from surface scattering data is proposed and mathematically justified. The method allows one to find small holes and cracks in metallic and other objects from the observation of the acoustic field scattered by the objects.
\end{abstract}

\section{INTRODUCTION}

In many applications one is interested in finding small inhomogeneities in a medium from the observation of the scattered field, acoustic or electromagnetic, on the surface of the medium.

We have two typical examples of such problems in mind. The first one is in the area of material science and technology. Suppose that a piece of metal or other material is given and one wants to examine if it has small cavities (holes or cracks ) inside. One irradiates the metal by acoustic waves and observes on the surface of the metal the scattered field. From these data one wants to determine:

1) are there small cavities inside the metal?

2 ) if there are cavities, then where are they located and what are their sizes?

Similar questions can be posed concerning localization not only of the cavities, but any small in comparison with the wavelength, inhomogeneities. Our methods allow one to answer such questions.

As a second example, we mention the mammography problem. Currently x-ray mammography is widely used as a method of early diagnistics of breast cancer in women. However, it is believed that the probability for a woman to get a new cancer cell in her breast as a result of an x-ray mammography test is rather high (about 35 percent). Therefore it is quite important to introduce ultrasound mammography tests. This is being done currently. A new cancer

* Department of Mathematics, Kansas State University, Manhattan, KS 66506-2602, USA. Email: ramm@math.ksu.edu 
cells can be considered as small inhomogeneities in the healthy breast tissue. The problem is to localize them from the observation on the surface of the breast of the scattered acoustic field.

The purpose of this short paper is to describe a new idea of solving the problem of finding inhomogeneities, small in comparison with the wavelength, from the observation of the scattered acoustic or electromagnetic waves on the surface of the medium.

For simplicity we present the basic ideas in the case of acoustic wave scattering. These ideas are based on the earlier results on wave scattering theory by small bodies, presented in [6-10]. Our objective in solving the inverse scattering problem of finding small inhomogeneities from surface scattering data are:

1) to develop a computationally simple and stable method for a partial solution to the above inverse scattering problem. The exact inversion procedures (see [9] and references therein) are computationally difficult and unstable. In practice it is often quite important, and sometimes sufficient for practical purposes, to get a "partial inversion", that is, to answer questions of the type we asked above: given the scattering data, can one determine if these data correspond to some small inhomogeneities inside the body? If yes, where are these inhomogeneities located? What are their intensities? We define the notion of intensity $v_{m}$ of an inhomogeneity below formula (1).

In Section 2 the basic idea of our approach is described. In Section 3 its short justification is presented. Some theoretical and numerical results based on a version of the proposed approach one can find in [6].

\section{BASIC EQUATIONS}

Let the governing equation be

$$
\left[\nabla^{2}+k^{2}+k^{2} v(x)\right] u=-\delta(x-y) \quad \text { in } \quad \mathbb{R}^{3}
$$

where $u$ satisfies the radiation condition, $k=$ const $>0$, and $v(x)$ is the inhomogeneity in the velocity profile.

Assume that $\sup _{x \in \mathbb{R}^{3}}|v(x)| \leq c_{0}, \operatorname{supp} v=U_{m=1}^{M} B_{m}\left(\tilde{z}_{m}, \rho_{m}\right) \subset \mathbb{R}_{-}^{3}=\{x \mid$ $\left.x_{(3)}<0\right\}$, where $x_{(3)}$ denotes the third component of vector $x$ in Cartesian coordinates, $B_{m}\left(\tilde{z}_{m}, \rho_{m}\right)$ is a ball, centered at $\tilde{z}_{m}$ with radius $\rho_{m}, k \rho_{m} \ll 1$.

Denote

$$
\bar{v}_{m}:=\int_{B_{m}} v(x) \mathrm{d} x .
$$

Inverse Problem (IP): given $u(x, y, k)$ for all $x, y \in P, P=\left\{x \mid x_{(3)}=0\right\}$ and a fixed $k>0$, find $\left\{\tilde{z}_{m}, \bar{v}_{m}\right\}, 1 \leq m \leq M$.

In this paper we propose a numerical method for solving the (IP).

To describe this method let us introduce the following notations:

$$
P:=\left\{x \mid x_{(3)}=0\right\}
$$




$$
\left\{x_{j}, y_{j}\right\}:=\xi_{j}, \quad 1 \leq j \leq J, \quad x_{j}, y_{j} \in P
$$

are the points at which the data $u\left(x_{j}, y_{j}, k\right)$ are collected

$$
k>0 \text { is fixed }
$$

$$
\begin{gathered}
g(x, y, k):=\frac{\exp (\mathrm{i} k|x-y|)}{4 \pi|x-y|} \\
G_{j}(z):=G\left(\xi_{j}, z\right):=g\left(x_{j}, z, k\right) g\left(y_{j}, z, k\right) \\
f_{j}:=\frac{u\left(x_{j}, y_{j}, k\right)-g\left(x_{j}, y_{j}, k\right)}{k^{2}} \\
\Phi\left(z_{1}, \ldots, z_{M}, v_{1}, \ldots, v_{M}\right):=\sum_{j=1}^{J}\left|f_{j}-\sum_{m=1}^{M} G_{j}\left(z_{m}\right) v_{m}\right|^{2} .
\end{gathered}
$$

The proposed method for solving the (IP) consists in finding the global minimizer of function (8). This minimizer $\left(\tilde{z}_{1}, \ldots, \tilde{z}_{M}, \tilde{v}_{1}, \ldots, \tilde{v}_{M}\right)$ gives the estimates of the positions $\tilde{z}_{m}$ of the small inhomogeneities and their intensities $\bar{v}_{m}$. This is explained in more detail below formula (14). Numerical realization of the proposed method, including a numerical procedure for estimating the number $M$ of small inhomogeneities from the surface scattering data is described in $[5]$.

Our approach with a suitable modification is valid in the situation when the Born approximation fails, for example, in the case of scattering by delta-type inhomogeneities [1].

In this case the basic condition $M k^{2} c_{0} \rho^{2} \ll 1(*)$ which guarantees the applicability of the Born approximation is violated. Here $\rho:=\max _{1 \leq m \leq M} \rho_{m}$ and $c_{0}$ was defined below formula (1). We assume throughout that $M$ is not very large, between 1 and 15 .

In the scattering by a delta-type inhomogeneity the assumption is $c_{0} \rho^{3}=$ const $:=V$ as $\rho \rightarrow 0$, so that for any fixed $k>0$ one has $k^{2} c_{0} \rho^{2}=k^{2} V \rho^{-1} \rightarrow \infty$ as $\rho \rightarrow 0$, and clearly condition $(*)$ is violated.

In our notations this delta-type inhomogeneity is of the form $k^{2} v(x)=$ $k^{2} \sum_{m=1}^{M} \bar{v}_{m} \delta\left(x-\tilde{z}_{m}\right)$.

The scattering theory by the delta-type potentials (see [1]) requires some facts from the theory of selfadjoint extensions of symmetric operators in Hilbert spaces and in this short paper we will not go into detail (see [4]).

\section{JUSTIFICATION OF THE PROPOSED METHOD}

We start with an exact integral equation equivalent to equation (1) with the radiation condition:

$$
u(x, y, k)=g(x, y, k)+k^{2} \sum_{m=1}^{M} \int_{B_{m}} g(x, z, k) v(z) u(z, y, k) \mathrm{d} z .
$$


For small inhomogeneities the integral on the right-hand side of (9) can be approximately written as

$$
\begin{aligned}
& k^{2} \int_{B_{m}} g(x, z, k) v(z) u(z, y, k) \mathrm{d} z:=k^{2} \int_{B_{m}} g(x, z, k) v(z) g(z, y, k) \mathrm{d} z+\varepsilon^{2} \\
& \quad=k^{2} G\left(x, y, \bar{z}_{m}\right) \int_{B_{m}} v \mathrm{~d} z+\varepsilon^{2}=k^{2} G\left(\xi, \bar{z}_{m}\right) \bar{v}_{m}+\varepsilon^{2}, \quad 1 \leq m \leq M
\end{aligned}
$$

where $\varepsilon^{2}$ is defined by the first equation in formula (10), it is the error due to replacing $u$ under the sign of integral in (9) by $g, \bar{z}_{m}$ is a point close to $\tilde{z}_{m}$.

One has $|u-g|=O\left(M k^{2} c_{0} \rho^{3} / d^{2}\right)$ if $x, y \in P$, so the error term $\varepsilon^{2}$ in (10) equals to $O\left(M^{2} k^{4} c_{0}^{2} \rho^{6} / d^{3}\right)$ if $x, y \in P$.

Therefore the function $u(z, y, k)$ under the sign of the integral in (9) can be replaced by $g(x, y, k)$ with a small error provided that

$$
c_{0} M \frac{k^{2} \rho^{3}}{d} \ll 1, \quad x, y \in P, \quad d \sim 1
$$

where $\rho=\max _{1 \leq m \leq M} \rho_{m}, c_{0}:=\max _{x \in \mathbb{R}^{3}}|v(x)|, M$ is the number of inhomogeneities, $d$ is the minimal distance from $B_{m}, m=1,2, \ldots, M$ to the surface $P$, $d \sim 1$ means that the length is measured in the units of length $d$.

If a sufficient condition for the validity of the Born approximation holds, that is,

$$
M k^{2} c_{0} \rho^{2}:=\delta \ll 1
$$

then

$$
O\left(\frac{M^{2} k^{4} c_{0}^{2} \rho^{6}}{d^{3}}\right)=O\left(\frac{\delta^{2} \rho^{2}}{d^{3}}\right) \ll 1 \quad \text { if } d \sim 1 .
$$

Note that $u$ in (9) has dimension $L^{-1}$, where $L$ is the length and $v(z)$ is dimensionless.

If the Born approximation is not valid, for example, if $c_{0} \rho^{3}=V \neq 0$ as $\rho \rightarrow 0$, which is the case of scattering by delta-type inhomogeneities, then the error term $\varepsilon^{2}$ in formula (10) can still be negligible: in this case $\varepsilon^{2}=O\left(M^{2} k^{4} V^{2} / d^{3}\right)$, so $\varepsilon^{2} \ll 1$ if $M^{2} k^{4} V^{2} / d^{3} \ll 1$.

If one understands a sufficient condition for the validity of the Born approximation as the condition which guarantees the smallness of $\varepsilon^{2}$ for all $x, y \in \mathbb{R}^{3}$ then condition (12) is such a condition. However, if one understands a sufficient condition for the validity of the Born approximation as the condition which guarantees the smallness of $\varepsilon^{2}$ for $x, y$ running only through the region where the scattered field is measured, in our case when $x, y \in P$, then a much weaker condition (11) will suffice.

In the limit $\rho \rightarrow 0$ and $c_{0} \rho^{3}=V \neq 0$ formula (10) takes the form (13) (see below). This can be derived from [1, p. 113]. Formula (1.1.33) in [1] shows that the resolvent kernel of the Schrödinger operator with the delta-type potential supported on a finite set of points (in our case on the set of points $\tilde{z}_{1}, \ldots, \tilde{z}_{M}$ ) 
has the form

$$
u(x, y, k)=g(x, y, k)+k^{2} \sum_{m=1}^{M} c_{m m^{\prime}} g\left(x, \tilde{z}_{m}\right) g\left(y, \tilde{z}_{m^{\prime}}\right)
$$

where $c_{m m^{\prime}}$ are some constants. These constants are determined by a selfadjoint realization of the corresponding Schrödinger operator with delta-type potential. There is an $M^{2}$-parametric family of such realizations (see [4] for more details).

Although in general the matrix $c_{m m^{\prime}}$ is not diagonal, under a practically reasonable assumption (11) one can neglect the off-diagonal terms of the matrix $c_{m m^{\prime}}$ and then formula (13) reduces practically to the form (10) with the term $\varepsilon^{2}$ neglected.

We have assumed in (10) that the point $\bar{z}_{m}$ exists such that

$$
\int_{B_{m}} g(x, z, k) v(z) g(z, y, k) \mathrm{d} z=G\left(x, y, \bar{z}_{m}\right) \bar{v}_{m} .
$$

This is an equation of the type of mean-value theorem. However, such a theorem does not hold, in general, for complex-valued functions. Therefore, if one wishes to have a rigorous derivation, one has to add to the error term $\varepsilon^{2}$ in (10) the error which comes from replacing of the integral $\int_{B_{m}} g(x, z, k) v(z) g(z, y, k) \mathrm{d} z$ in (10) by the term $G\left(x, y, \bar{z}_{m}\right) \bar{v}_{m}$. The error of such an approximation can be easily estimated. We do not give such an estimate, because the basic conclusion that the error term is negligible compared with the main term $k^{2} G\left(x, y, \bar{z}_{m}\right) \bar{v}_{m}$ remains valid under our basic assumption $k \rho \ll 1$. From (10) and (7) it follows that

$$
f_{j} \approx \sum_{m=1}^{M} G_{j}\left(\bar{z}_{m}\right) \bar{v}_{m}, \quad G_{j}\left(\bar{z}_{m}\right):=G\left(\xi_{j}, \bar{z}_{m}, k\right) .
$$

Therefore, parameters $\tilde{z}_{m}$ and $\bar{v}_{m}$ can be estimated by the least-squares method if one finds the global minimum of the function (8):

$$
\Phi\left(z_{1}, \ldots, z_{M}, v_{1}, \ldots, v_{M}\right)=\min
$$

Indeed, if one neglects the error of the approximation (10), then the function (8) is a smooth function of several variables, namely, of $z_{1}, z_{2}, \ldots, z_{M}, v_{1}, v_{2}, \ldots, v_{M}$, and the global minimum of this function is zero and is attained at the actual intensities $\bar{v}_{1}, \bar{v}_{2}, \ldots, \bar{v}_{M}$ and at the values $z_{i}=\bar{z}_{i}, i=1,2, \ldots, M$.

This follows from the simple argument: if the error of approximation is neglected, then the approximate equality in (14) becomes an exact one. Therefore $f_{j}-\sum_{m=1}^{M} G_{j}\left(\bar{z}_{m}\right) \bar{v}_{m}=0$, so that function (8) equals to zero. Since this function is non-negative by definition, it follows that the values $\bar{z}_{m}$ and $\bar{v}_{m}$ are global minimizers of the function (8). Therefore we take the global minimizers of function (8) as approximate values of the positions and intensities of the small inhomogeneities. 
In general we do not know that the global minimizer is unique. For the case of one small inhomogeneity $(m=1)$ uniqueness of the global minimizer is proved in [6] for all sufficiently small $\rho_{m}$ for a problem with a different functional. The problem considered in [6] is the (IP) with $M=1$, and the functional minimized in $[6]$ is specific for one inhomogeneity.

The scattering theory for small scatterers originated in the classical works of Lord Rayleigh. It was developed in [7] and [8], where analytical formulas for the scattering matrix were derived for the acoustic and electromagnetic scattering problems. In [7] and [9] inverse scattering problems for small bodies are considered. Numerically an important ingredient of our approach is the solution of the global minimization problem (14). The theory of global minimization is developed extensively and the literature of this subject is quite large. We mention two recent papers [2] and [3] where the reader can find many references.

\section{REFERENCES}

1. S. Albeverio, F. Gesztesy, R. Hoegh-Krohn, H. Holden, Solvable Models in Quantum Mechanics. Springer Verlag, New York, 1988.

2. J. Barhen and V. Protopopescu, Generalized TRUST algorithms for global optimization. In: State of the Art in Global Optimization (Eds C. Floudas and P. Pardalos). Kluwer Acad., Boston, 1996, 163-180.

3. J. Barhen, V. Protopopescu, and D. Reister, TRUST: A deterministic algorithm for global optimization. Science (1997) 276, 1094-1097.

4. F. Gesztesy and A. G. Ramm, An inverse problem for point inhomogeneities. Methods of Functional Analysis and Topology (2000) 6, No. 2.

5. S. Gutman and A. G. Ramm, Application of the hybrid stochastic-deterministic minimization method to a surface data inverse scattering problem. In: Operator Theory and its Applications

(Eds A. G. Ramm, P. N. Shivakumar, and A. V. Strauss). Amer. Math. Soc., Fields Institute Communications Vol. 25, Providence, 2000, 293-304.

6. A. I. Katsevich and A. G. Ramm, Approximate inverse geophysical scattering on a small body. SIAM J. Appl. Math. (1996) 56, No. 1, 192-218.

7. A. G. Ramm, Iterative Methods for Calculating the Static Fields and Wave Scattering by Small Bodies. Springer Verlag, New York, 1982.

8. A. G. Ramm, Scattering by Obstacles. D. Reidel, Dordrecht, 1986.

9. A. G. Ramm, Multidimensional Inverse Scattering Problems. Longman/Wiley, New York, 1992.

10. A. G. Ramm, A method for finding small inhomogeneities from surface data. Math. Sci. Research Hot-Line (1997) 1, No. 10, 40-42. 